\title{
USO DE GEOTECNOLOGIAS NA SELEÇÃO DE ÁREAS PARA IMPLANTAÇÃO DE ATERROS SANITÁRIOS: ABORDAGEM COMPOSTA APLICADA AO MUNICÍPIO DE MIRANDÓPOLIS, SP
}

\author{
AREA SELECTION FOR LANDFILL INSTALLATION USING A COMPOSED APPROACH: CASE \\ STUDY IN MIRANDÓPOLIS, SÃO PAULO STATE, BRAZIL
}

\section{Mariana Barbosa de CARVALHO; Nelson GIOVANINI JUNIOR; José Augusto de LOLLO; César Gustavo da Rocha LIMA}

Universidade Estadual Paulista Júlio de Mesquita Filho - Campus de Ilha Solteira - Departamento de Engenharia Civil. E-mails: maa.carvalho@ hotmail.com; jose.lollo@unesp.br; nelsongiovanini@ outlook.com; cesar.lima@ dec.feis.unesp.br

\author{
Introdução \\ Materiais e Métodos \\ Área de Estudo \\ Materiais \\ Procedimentos Técnicos \\ Resultados e Discussões \\ Conclusões \\ Referências
}

RESUMO - O elevado crescimento populacional e o consequente aumento da produção de resíduos sólidos têm sido motivo de preocupação no gerenciamento e disposição desses materiais, especialmente devido à constante diminuição de áreas ambientalmente adequadas e logisticamente viáveis para a implementação de aterros sanitários. Para a seleção do local de instalação de um aterro sanitário devem ser considerados vários aspectos técnicos, legais e ambientais, que se não forem corretamente definidos podem resultar em problemas para a administração pública, para a população, e para o meio ambiente. Dessa forma, é de interesse geral que sejam elaborados métodos para simplificar a tomada de decisão quanto à instalação desses depósitos de resíduos sólidos mantendo a eficiência do processo de análise. Este trabalho desenvolveu uma análise de decisão multicritério composta pela associação da ponderação de atributos ambientais, restritivos (Legais) e logísticos para pré-seleção de áreas aptas à instalação de um aterro sanitário no município de Mirandópolis - SP, fazendo-se uso dos Sistema de Informações Geográficas (SIG), aplicação da lógica Fuzzy e métodos de ponderação algébrica de mapas. Na análise, foram primeiramente considerados critérios restritivos, dividindo a área do município em parcelas aptas e não aptas; posteriormente, com base em parâmetros e ponderações encontrados na literatura, realizou-se a classificação das áreas aptas para identificar quais seriam as mais adequadas à instalação do aterro. Verificou-se que menos de $40 \%$ da área total do município de Mirandópolis-SP é apta para a instalação de aterro sanitário, e que as áreas mais adequadas se encontram no entorno do perímetro urbano, especialmente a nordeste e sudoeste deste. A principal vantagem da abordagem adotada é ter sua base em dados digitais disponíveis e de fácil acesso público, o que, associado a técnicas digitais de análise espacial, proporcionou agilidade ao processo. A natureza objetiva da abordagem utilizada permite sua aplicação a outros municípios, tornando-a uma ferramenta com grande potencial para a administração pública municipal na pré-seleção de áreas para instalação de aterros sanitários.

Palavras-chave: Aterro sanitário, Logística, Meio ambiente, SIG.

\begin{abstract}
The high population growth and the consequent increase in waste production have been a concern in the management and disposal of material, especially due to the constant reduction of environmentally adequate and logistically viable areas for the implementation of landfills. For the area selection various technical, legal and environmental aspects must be considered, which, if not correctly defined, can result in problems for the public administration, for the population, and for the environment. Thus, it is of general interest to develop methods to simplify decision making regarding the installation of solid waste deposits while maintaining the efficiency of the analysis process. This work developed a multicriteria decision analysis composed by the association of the weighting of environmental attributes, restrictive attributes (Legal) and logistic attributes for the pre-selection of suitable areas for the installation of a sanitary landfill in the city of Mirandópolis - SP, making use of Geographic Information System (GIS), application of Fuzzy logic, and weighting methods using algebra of maps. In the analysis, restrictive criteria were first considered, dividing the area of the county into suitable and unsuitable plots, later was conducted the classification of the suitable areas to identify which would be the most adequate to the installation of the landfill, based on parameters and weights found in the literature. It was verified that less than $40 \%$ of the total area of the municipality is suitable for the installation of landfills, and that the most adequate areas are located around the urban perimeter, especially to the northeast and southwest of it. The main advantage of the method adopted is to have its digital database easily available and publicly accessible, which, combined with digital techniques of spatial analysis, provided agility to the process. The objective of the used approach allows its application in other municipalities, making it a tool with potential usability for municipal public administration.
\end{abstract}

Keywords: Landfill, Logistics, Environment, GIS.

\section{INTRODUÇÃOO}

O gerenciamento de resíduos sólidos dos municípios é, atualmente, uma das maiores preocupações governamentais, principalmente devido ao aumento populacional e o conse-

quente incremento na geração de lixo, bem como, devido à redução de áreas ambiental-mente aptas e logisticamente viáveis para a implantação de depósitos permanentes desses materiais. Bing et 
al. (2016) reforçam que a logística do gerenciamento dos resíduos sólidos municipais é necessária para atender o crescente volume gerado e para a implantação de políticas de reutilização de materiais não renováveis, propiciando a diminuição de impactos ambientais, consumo de energia e gastos de recursos financeiros (Eriksson et al., 2005).

Segundo Hoornweg \& Bhada-Tata (2012), estima-se que a geração mundial de resíduos sólidos irá dobrar até o ano de 2025, passando de 1.3 bilhões de toneladas/ano para 2.2 bilhões de toneladas de resíduos. Tal previsão, de certa forma, gera uma tendência no estudo do gerenciamento dos resíduos sólidos em avaliar os possíveis impactos da disposição desse material (Raghavan et al., 2015). Assim, para o gerenciamento dos resíduos sólidos urbanos são observadas em todo o planeta abordagens sustentáveis para a sua disposição adequada (Lella et al., 2017).

De acordo com Deus et al. (2017), no Brasil, mesmo com a presença de uma Política Nacional de Resíduos Sólidos (BRASIL, 2010), dados gerais sugerem um inadequado gerenciamento desses resíduos (SÃO PAULO, 2013, 2014). Um exemplo disso pode ser relacionado com a reciclagem, que, mesmo sendo um tipo de tratamento comprovadamente eficaz, é raramente implementado com sucesso nos municípios brasileiros.

Ainda de acordo com Ferreira et al. (2017), mesmo onde há uma melhora na capacidade municipal em gerenciar a coleta de resíduos, um dos mais recorrentes problemas diz respeito aos locais da instalação dos aterros sanitários. Muitas vezes, não há nenhum critério técnico na escolha da área de instalação e funcionamento, provocando divergências com as normas legais vigentes. Tal fato pode estar diretamente ligado à falta de mecanismos técnico operacionais de fácil acesso e desenvolvimento pelos órgãos públicos responsáveis.

Assim, são facilmente encontradas situações irregulares, as quais poderiam ser evitadas por meio de estudos prévios com foco na situação e especificidades ambientais de cada local, buscando otimizar a instalação do aterro sanitário conforme legislação vigente.

Selecionar locais propícios à instalação de aterros sanitários é um processo complexo, pois é necessária uma avaliação multidisciplinar que incorpora informações naturais, sociais, científicas, políticas, legais, éticas entre outras. Uma vez que aterros sanitários são popularmente vistos como ameaça à saúde pública, à qualidade de vida humana e dos ecossistemas em seu entorno, eles passam a pertencer a um grupo de instalações indesejadas pela sociedade (Kikuchi \& Gerardo, 2009).

Com relação à identificação de áreas ambientalmente aptas para acomodar um aterro sanitário, é pertinente admitir que os principais impactos potenciais gerados pela atividade em questão são: a contaminação do solo, subsolo e das águas superficiais e sub-superficiais; a geração de gases e de odores; e a possibilidade de intensificação de processos erosivos devido à intensa movimentação de terra (Montaño et al., 2012).

Há ainda fatores antrópicos e socioeconômicos que são mais subjetivos e difíceis de mensurar uma vez que são intrínsecos ao local, variando com a economia ou cultura do local, podendo ou não estar presentes. Alguns desses fatores são: alterações do tráfego de veículos em área rural, mudança de paisagem, desvalorização de terras ao redor do local, movimentação e aparecimento de populações carentes, problemas de política pública, problemas sanitários e diversos outros (Montaño et al., 2012; Feo \& Gisi, 2014; Gorsevski et al., 2012).

Geralmente, o processo de locação de um aterro pode ser dividido em três fases (Eastman et al., 1995; Feo \& Gisi, 2014). Na primeira (análise macro de locação) o objetivo é selecionar "áreas não aptas" assim como "áreas potencialmente aptas" tendo como base o critério de exclusão obtidos através da literatura e/ou legislações. O critério de exclusão significa a não seleção daquela área para locação do aterro.

A segunda fase (micro locação e análise espacial) é focada em identificar uma lista de locais no espaço de estudo com o uso de critérios de preferência e penalização. $\mathrm{O}$ critério de preferência indica a presença de elementos de aptidão assim como de conveniência para o local.

O critério de penalidade atribui a presença de contraindicações sugerindo que a construção naquele local seja feita apenas tomando extremos cuidados. $\mathrm{O}$ critério de penalização será discriminatório e não excludente. Já a última etapa inclui a seleção da área mais susceptível entres as áreas potenciais (Feo \& Gisi, 2014).

Uma das ferramentas de apoio que pode ser utilizada para tratamento de dados espaciais são os Sistemas de Informações Geográficas (SIGs), que tornam possível a avaliação simultânea de diversos critérios técnicos (Camara et al., 1996), 
inclusive podendo ser muito útil para a escolha de áreas aptas à instalação de aterros sanitários, facilitando assim a seleção de áreas mais adequadas.

Num processo de decisão multicritério, os fatores de decisão são ponderados segundo pesos que melhor representam a importância relativa dos atributos na análise. O processo analítico hierárquico (AHP) e a lógica Fuzzy são as duas modalidades técnicas mais utilizadas em análise multicritério

O processo AHP (Saaty, 1977), é um método no qual são aplicadas comparações pareadas de diferentes parâmetros atribuindo pesos relativos a cada informação (Khan et al., 2018). A sua aplicação demanda um amplo conhecimento dos critérios envolvidos, uma vez que implica encontrar a contribuição/peso de cada um deles no conjunto. Por vezes os gestores públicos, principalmente em pequenos municípios, não possuem tal conhecimento. Em tais condições, pode se lançar mão de experiências anteriores tais como Schmidt (2017), Spigolon et al. (2015), Motlagh \& Sayadi (2015), Feo \& Gisi (2014), entre outros. Outro método de elevado potencial segundo Eastman (2005) é a lógica nebulosa ou lógica Fuzzy, na qual são atribuídos valores parciais entre a não aceitação e a aceitação de um parâmetro. A integração entre modelos multicritério, a lógica nebulosa e os processos dos SIGs utilizam, basicamente, dados espaciais e critérios de peso relativo para produzir informações georreferenciadas de maior confiança para serem utilizadas em tomadas de decisão.

Assim, diante do exposto, o presente trabalho objetivou elaborar um processo de decisão multicritério composta pela associação da ponderação de atributos ambientais, restritivos (Legais) e logísticos de fácil implementação pelos órgãos públicos utilizando análise espacial e aplicação da lógica Fuzzy para avaliar a melhor locação de um aterro sanitário, tendo como estudo de caso o município de Mirandópolis, SP.

\section{MATERIAIS E MÉTODOS}

\section{Área de Estudo}

O presente trabalho foi desenvolvido tendo como estudo de caso o Município de Mirandópolis, localizado na porção noroeste do Estado de São Paulo, microrregião de Andradina (Figura 1). O município possui uma área de aproximadamente $918 \mathrm{~km}^{2}$, delimitado pelo quadrante geográfico dado pelas seguintes coordenadas geográficas $21^{\circ} 21^{\prime} 45^{\prime \prime} \mathrm{S}-51^{\circ} 21^{\prime} 45^{\prime \prime} \mathrm{O}$ e $20^{\circ} 47^{\prime} 49^{\prime \prime} \mathrm{S}$ $50^{\circ} 56^{\prime} 24$ '”.

O município de Mirandópolis possui uma população estimada de aproximadamente 29.315 (IBGE, 2017). As suas principais atividades econômicas desenvolvidas estão relacionadas com a prestação de serviços (predominante), indústria, e também ligadas ao setor agropecuário. A área rural do município é coberta em sua maior parte por pastagens, com uma área de 444,48 $\mathrm{km}^{2}$, seguido por lavouras permanentes ou temporárias ocupando uma área de $97,33 \mathrm{~km}^{2}$ (IBGE, 2017). Entre as lavouras há plantações de cana-de-açúcar em grandes fazendas e diversos outros tipos de cultivos em pequenas propriedades como o abacaxi, goiaba e acerola.

A coleta de resíduos sólidos no município alcança 99,53\% das residências (SEADE, 2017) e dispõe de coleta seletiva para reciclagem e de resíduos da construção civil (SNIS, 2016). Apesar de quase que a totalidade das residências serem atendidas pela coleta, o município ainda não possui um local adequado de destinação final dos resíduos sólidos urbanos (RSU), e todo esse material gerado está sendo disposto no município de VotuporangaSP (distante cerca de $200 \mathrm{~km}$ ), elevando os gastos municipais.

\section{Materiais}

Os materiais utilizados como fonte de dados para a realização do presente estudo foram:

- Base digital de dados regionais fornecidos pela Prefeitura Municipal de Mirandópolis-SP (SAAEM, 2014) contendo: malha viária, hidrografia, limites do município e do perímetro urbano, localização de poços rurais de abastecimento hídrico, topografia e, características do solo;

- Dados do Modelo Digital de Elevação do satélite ALOS, Sensor Palsar (JAXA, 2018), com resolução espacial de 12,5 metros;

- Imagem de satélite CBERS 4, sensor MUX (resolução espacial de 20,0 m), bandas 6-B, 7-G, 8$\mathrm{R}$, quadrantes 123 e 124, de 12/08/2017 (INPE, 2017);

- Dados do Sistema Nacional de Cadastro Ambiental Rural (SICAR, 2017), contendo áreas de vegetação nativa, reservas legais e áreas de preservação permanente (APPs). 


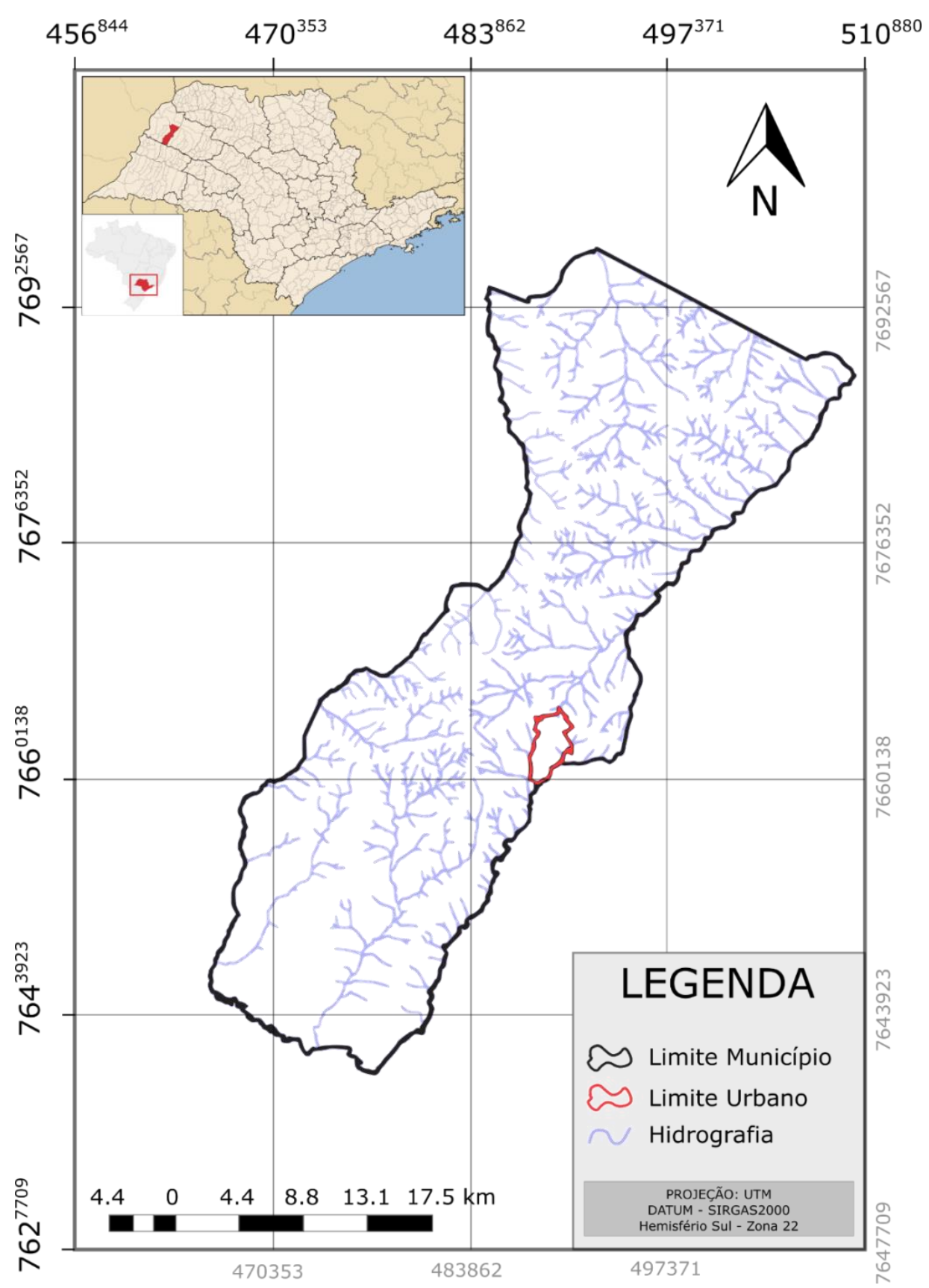

Figura 1 - Localização da área de estudo (município de Mirandópolis-SP).

\section{Procedimentos Técnicos}

A criação do banco de dados geográfico, bem como todos os procedimentos técnicos foram desenvolvidos no sistema SPRING 5.5.1 (INPE, 2018).

Para a condução do estudo, o banco de dados geográficos foi criado tendo como base os seguintes planos de informações (PIs) da área de estudo: limite do município, perímetro urbano, residências isoladas/núcleos popula-cionais, malha viária, área de servidão (dutos/gasodutos), hidrografia, poços de abastecimento hídrico, área de preservação permanente (APP), reserva legal, tipos de solos, altimetria, ocupação do solo. Deve-se observar que em função das diferentes resoluções dos materiais originais utilizados, e tendo em vista o agrupamento dos planos de informação por meio da álgebra de mapas, todos os mapeamentos finais produzidos no presente estudo foram remostados com resolução espacial de $10 \times 10 \mathrm{~m}$, compatível com escala 1:25.000.

A metodologia detalhada, bem como todas suas etapas podem ser verificadas na figura 2 .

Com base nos dados já descritos, foram adotadas diversas abordagens de natureza legal e técnica para a geração dos mapas temáticos intermediários utilizados na geração do mapa de aptidão para instalação de aterros sanitários. Assim, no presente estudo foram considerados, a princípio, os parâmetros restritivos para uma primeira aproximação na determinação de áreas aptas e não aptas, de acordo com a lógica booleana. Cada 
restrição foi baseada em uma afirmação ou negação de informações limitando a análise a uma região especifica conforme a necessidade de estudo. Essas informações foram observadas nas legislações federal e estadual paulista, normas e demais estudos técnicos vigentes, conforme a tabela 1 .

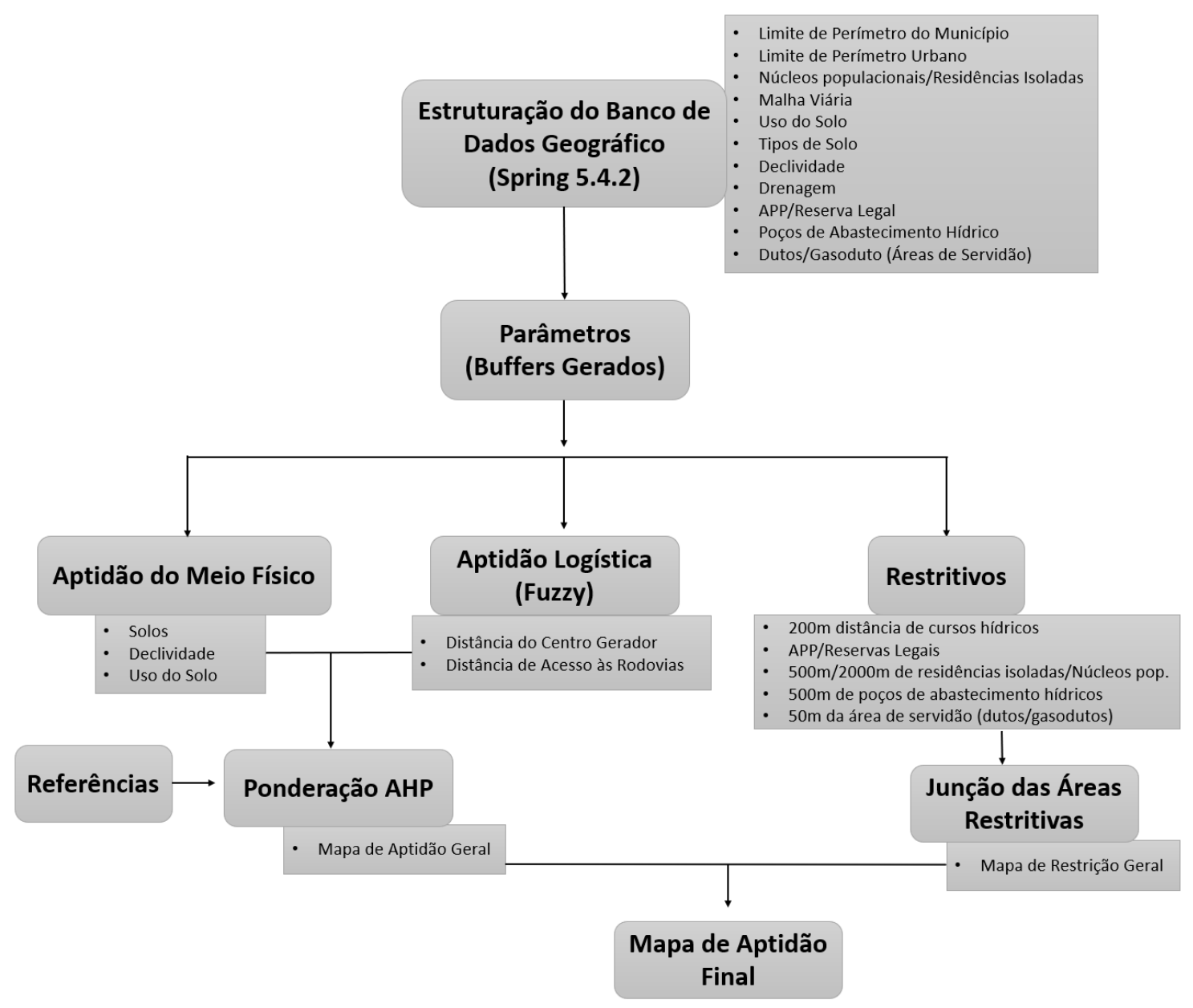

Figura 2 - Representação das etapas metodológicas realizadas.

Ressalta-se que no caso específico da permeabilidade do solo, bem como da profundidade do nível freático (Tabela 1), por se tratarem de informações geralmente indisponíveis em bancos de dados públicos, por serem de aquisição onerosa em campo para grandes áreas como a do município de Mirandópolis, tais avaliações podem ser feitas a campo, de modo reduzido, como critério final restritivo apenas entre as possíveis áreas que forem caracterizadas como aptas.

Assim, a partir das restrições apresentadas na tabela 1, gerou-se o primeiro conjunto de buffers por meio da ferramenta "mapa de distância" do software Spring correspondentes aos poços/gasodutos (Figura 3a), núcleos populacionais (Figura 3b) e corpos hídricos (Figura 3c). Para o critério restritivo referente às áreas de preservação permanente e reservas legais foram utilizados os dados do Sistema Nacional de Cadastro Ambiental Rural
(SICAR, 2017), conforme apresentado na figura $3 \mathrm{~d}$.

Em seguida foram considerados os parâmetros que estabelecem graus de importância para as áreas aptas. Nesse caso, os parâmetros utilizados foram: a) de aptidão do meio físico (tipo de solo, uso do solo e declividade); b) de aptidão logística (distância até rodovias e distância até o centro de coleta).

Para geração dos parâmetros de aptidão do meio físico, os mapas foram elaborados conforme critérios descrito na tabela 2.

Assim, o mapa de solos (Figura 4a) foi extraído a partir dos dados municipais (SAAEM, 2014), ao passo que o mapa de declividade (Figura 4c) foi extraído tendo como base os dados de modelo digital de elevação do Satélite Alos (JAXA, 2018) os quais foram tratados dentro do sistema Spring por meio das operações "geração de declividade" e "fatiamento" conforme as classes desejadas. 
Tabela 1 - Parâmetros restritivos observados.

\begin{tabular}{|c|c|c|c|}
\hline Parâmetro & Fonte & Restrição $^{(a)}$ & Observações \\
\hline $\begin{array}{l}\text { Topografia } \\
\text { (declividade) }\end{array}$ & SÃO PAULO (2005) & $\begin{array}{l}\text { *Não são recomendáveis } \\
\text { áreas maior que } 10 \%\end{array}$ & $\begin{array}{l}\text { As áreas não devem ter } \\
\text { características muito inclinadas. } \\
\text { Áreas muito planas representam } \\
\text { maior tendência de infiltração. }\end{array}$ \\
\hline Tipo de solo & ABNT (1997) & $\begin{array}{l}* * \text { Permeabilidades } \\
\text { superiores a } 10^{-6} \mathrm{~cm} / \mathrm{s} \text { são } \\
\text { restritivas }\end{array}$ & $\begin{array}{l}\text { Área com solos homogêneos de } \\
\text { argila e alguns tipos de siltes são } \\
\text { desejáveis. }\end{array}$ \\
\hline $\begin{array}{l}\text { Distância aos } \\
\text { corpos de água }\end{array}$ & $\begin{array}{l}\text { SÃO PAULO (2005) } \\
\text { ABNT (1997) }\end{array}$ & $\begin{array}{l}\text { Mínimo de } 200 \text { m de } \\
\text { qualquer corpo hídrico }\end{array}$ & - \\
\hline $\begin{array}{l}\text { Profundida de nível } \\
\text { freático }\end{array}$ & $\begin{array}{l}\text { SÃO PAULO (2005) } \\
\text { ABNT (1997) }\end{array}$ & **Mínimo de 3,00 metros & $\begin{array}{l}\text { Para solos arenosos são } \\
\text { interessantes maiores } \\
\text { profundidades. }\end{array}$ \\
\hline $\begin{array}{c}\text { Distância de núcleo } \\
\text { populacionais }\end{array}$ & SÃO PAULO (2005) & $\begin{array}{l}\text { Mínimo de } 500 \text { metros de } \\
\text { residências isoladas e } 2000 \\
\text { metros de núcleos urbanos }\end{array}$ & - \\
\hline $\begin{array}{l}\text { APPs e Reservas } \\
\text { Legais }\end{array}$ & $\begin{array}{l}\text { SÃO PAULO (2005) } \\
\text { ABNT (1997) }\end{array}$ & Presença indica área inapta & $\begin{array}{c}\text { Em alguns casos as matas podem } \\
\text { ser atenuantes de interferências } \\
\text { negativas }\end{array}$ \\
\hline Poços de água & Chang et al. (2008) & $\begin{array}{l}\text { Mínimo de } 500 \text { metros de } \\
\text { poços }\end{array}$ & $\begin{array}{c}\text { Semelhante a residência isolada } \\
\text { de acordo com a legislação do } \\
\text { estado }\end{array}$ \\
\hline Gasoduto & TBG (2017) & $\begin{array}{c}50 \text { metros após a faixa de } \\
\text { servidão que possui } 20 \\
\text { metros }\end{array}$ & - \\
\hline
\end{tabular}

(a) * = Recomendações técnicas, porém não via de regra em função das características de relevo de cada município/região estudada, ${ }^{* *}=$ Avaliação final deve ser realizada em campo.

Para o uso e ocupação do solo (Figura 4b) foi realizada uma classificação supervisionada por pixel (MAXVER) e posterior pós-classificação manual, elaborada a partir de imagens de satélite CBERS 4 (12/08/2017), utilizando-se da seguinte composição das bandas $5(\mathrm{~B}), 6(\mathrm{G})$ e $7(\mathrm{R})$. No processo de pós-classificação, visando localizar e corrigir possíveis inconsistências, utilizou-se do auxílio de imagens de alta resolução do Google Earth Pro (Sullivan, 2009).

Considerando as características agropecuárias do município, e considerando a não existência de critérios técnicos que distinguem efeitos de diferentes culturas no solo para essa finalidade, as classes adotadas foram: vegetação de grande porte e outros usos conforme Feo \& Gisi (2014).

No caso da aptidão logística, utilizou-se uma escala de valores contínuos por meio de operadores Fuzzy (funções descritas nas equações 1 e 2) para determinação das classes dos parâmetros "distância até rodovias" e de "distância até o centro de coleta", tendo como parâmetro as ponderações descritas na tabela 3.

A lógica Fuzzy foi conduzida de forma a estabelecer uma ponderação contínua para os fatores de distância, e então esses valores foram somados às ponderações referentes aos outros parâmetros.

Assim, foi feita uma avaliação das vantagens e desvantagens de cada área definida como apta após o processo de eliminação de locais não permitidos para a instalação do aterro.

Deste modo, baseado na lógica Fuzzy foram definidas as funções para obtenção do "Valor" para cada ponto de uma grade numérica conforme a distância " $\mathrm{D}$ " (do centro gerador e das rodovias). As funções aplicadas foram:

1) Para distâncias a partir do centro geométrico de coleta (gerado a partir do vetor do limite do perímetro urbano - Figura 5b):

$$
\text { Valor }_{\text {urb }}=3,75-0,000375 \times D_{\text {urb }}
$$

onde: $\mathrm{D}_{\text {urb }}=$ distância em metros a partir do limite do perímetro urbano, $\mathrm{e}$

2) Para distância a partir da malha viária (Figura 5a):

$$
\text { Valor }_{\text {rod }}=3,11688312-0,00155844 \times \mathrm{D}_{\text {rod }} \text { (2) }
$$

onde: $\mathrm{D}_{\text {rod }}=$ distâncias em metros a partir dos vetores que representam a malha viária. 

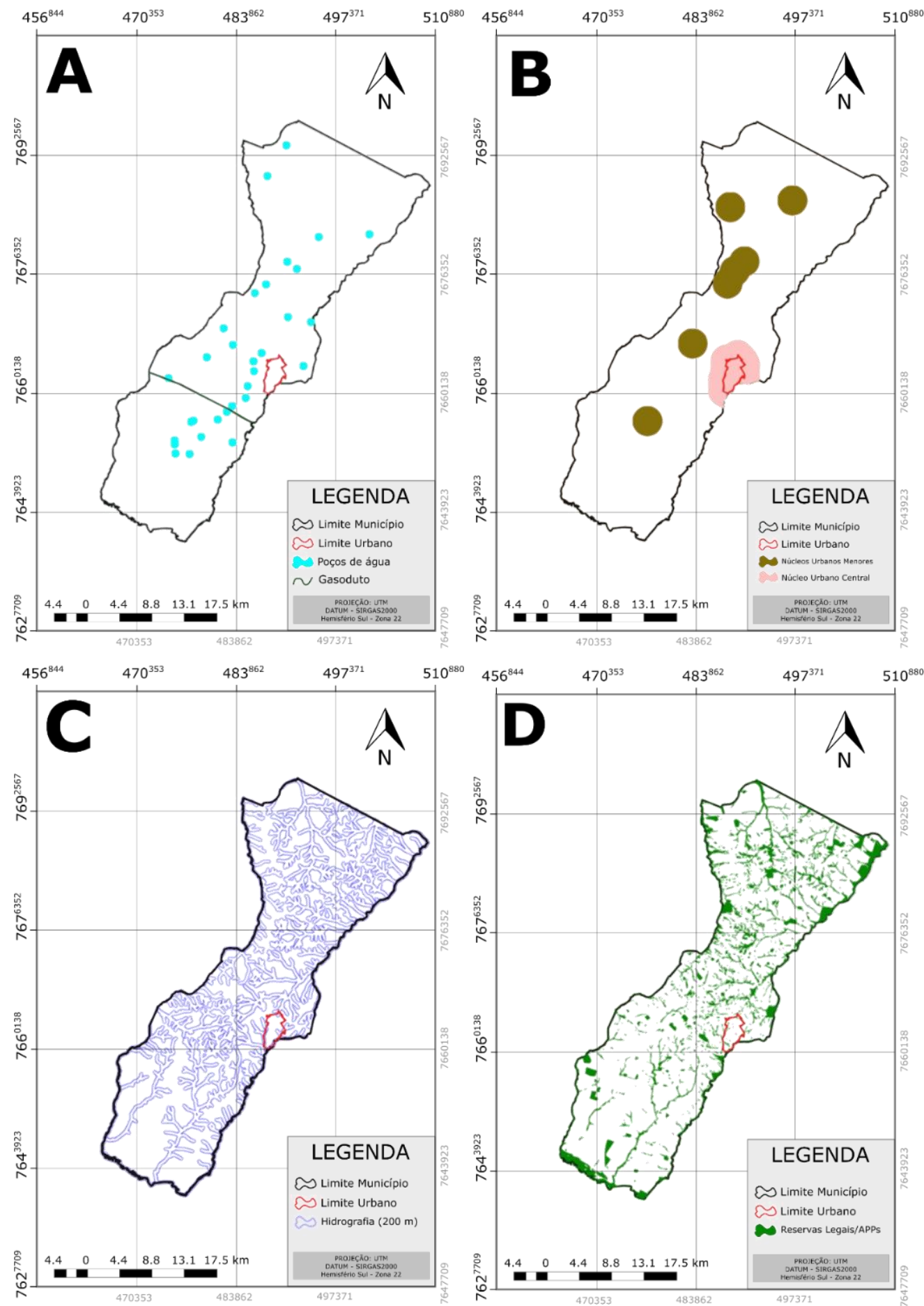

Figura 3 - Buffers referentes às áreas restritivas para o município de Mirandópolis-SP, onde, (A) poços de água; (B) Distância de núcleos populacionais; (C) Distância de corpos de água e (D) APPs e reservas legais.

Tabela 2 - Parâmetros de aptidão do meio físico.

\begin{tabular}{|c|c|c|c|c|}
\hline \multirow{2}{*}{ Fatores } & \multirow{2}{*}{ Fonte } & \multicolumn{3}{|c|}{ Aptidão } \\
\hline & & Baixa & Média & Alta \\
\hline Tipo de solo & Montaño et al. (2012) & $\begin{array}{c}\text { Neossolo litólico e } \\
\text { quartzarênico }\end{array}$ & $\begin{array}{c}\text { Latossolo vermelho- } \\
\text { amarelo; Argissolos } \\
\text { e Nitossolos } \\
\end{array}$ & $\begin{array}{l}\text { Latossolos eutroférrico, } \\
\text { distroférrico e vermelho }\end{array}$ \\
\hline Uso do solo & Feo \& Gisi (2014) & $\begin{array}{c}\text { Vegetação arbórea } \\
\text { de grande porte }\end{array}$ & - & Outros usos \\
\hline Declividade & Montaño et al. (2012) & - & Inferior a $2 \%$ & Entre $2 \%$ e $10 \% *$ \\
\hline
\end{tabular}

* Limite superior definido conforme disposto em SÃO PAULO (2005). 

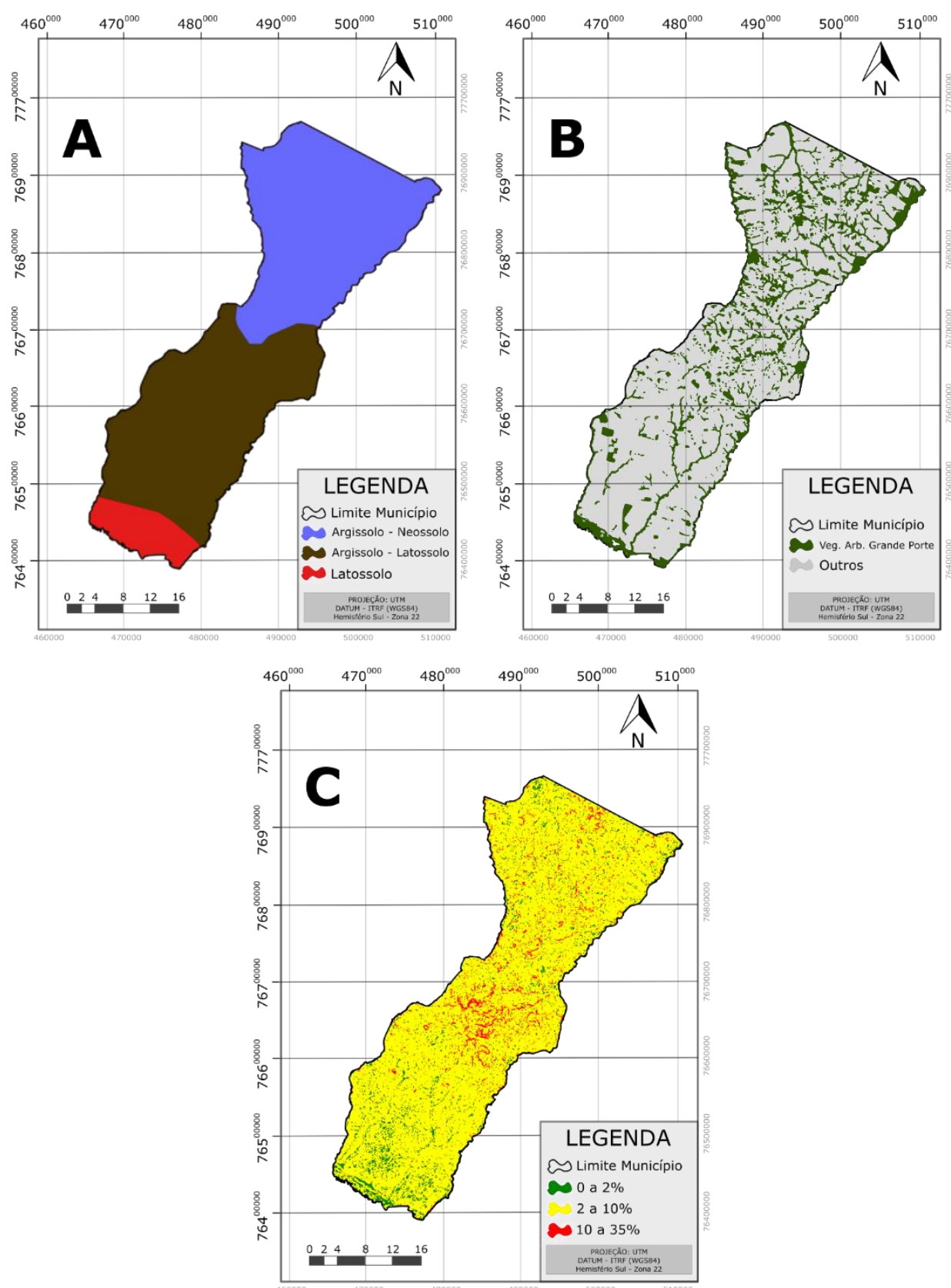

Figura 4 - Mapas de aptidão do meio físico para o município de Mirandópolis-SP, onde (A) refere-se ao tipo de solo; (B) Uso do solo e (C) Níveis de declividade.

Tabela 3 - Parâmetros de aptidão logística.

\begin{tabular}{c|c|c|c}
\hline \multirow{2}{*}{ Fatores } & Fontes & Mínimo & Máximo \\
\cline { 2 - 4 } & Chang et al. (2008) & $75 \mathrm{~m}$ & $2.000 \mathrm{~m}$ \\
\hline Distância até rodovias & Kahraman et al. (2018) & $2.000 \mathrm{~m}$ & $10.000 \mathrm{~m}$ \\
\hline $\begin{array}{c}\text { Distância até centro geométrico } \\
\text { de coleta }\end{array}$ & & \\
\hline
\end{tabular}

As funções de distância foram encontradas a partir da equação da reta na qual a maior aptidão (considerada como o valor 3, sendo este o mesmo valor atribuído às classes de alta aptidão para os parâmetros absolutos) refere-se à distância mínima permitida, enquanto a menor aptidão (valor zero) refere-se à distância máxima.

A partir de tais grades numéricas foram gerados os mapas temáticos dos limites das classes dos parâmetros logísticos (Figura 5a, b), os quais no presente caso ilustram apenas os dados de transição entre as classes, e não a variação gradual que as distâncias receberam por meio dos operadores Fuzzy. Para estabelecer a relação de importância entre os critérios utilizados, foi realizada uma análise matemática a partir de outros trabalhos de mesmo tema que fizeram uso de diferentes métodos de ponderação multicritério, 
especialmente do método AHP (Analytic Hierarchy Process). A ponderação foi feita a partir da média entre os valores encontrados na literatura pesquisada, sendo posteriormente os valores das

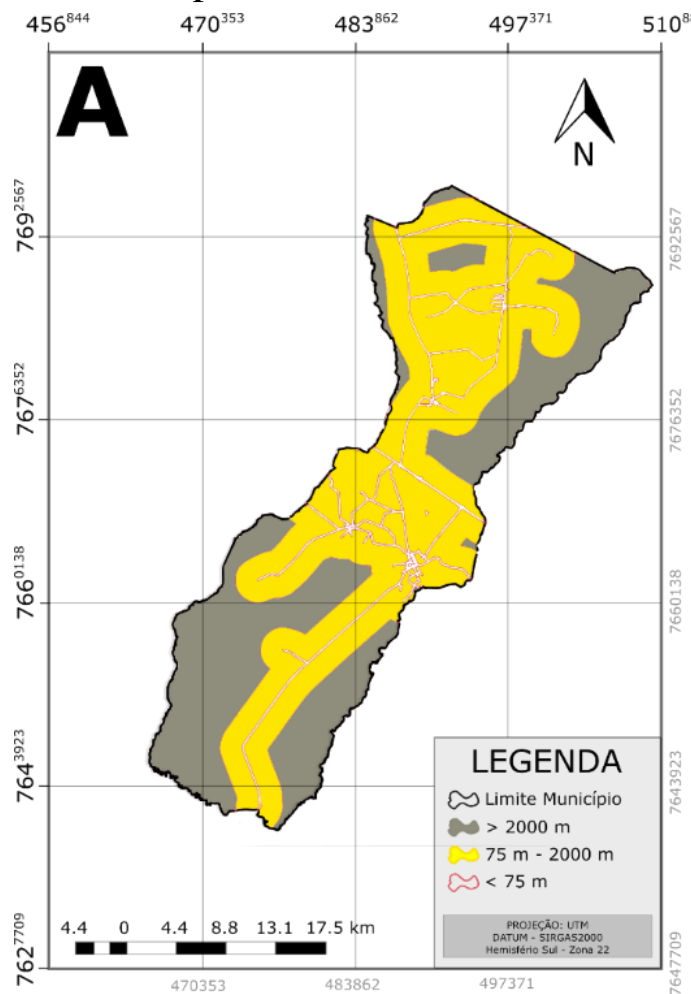

médias normalizados de forma a representarem uma distribuição de pesos cuja soma é $100 \%$. A tabela 4 apresenta os dados de compilação de dados, as médias brutas e as médias normalizadas.

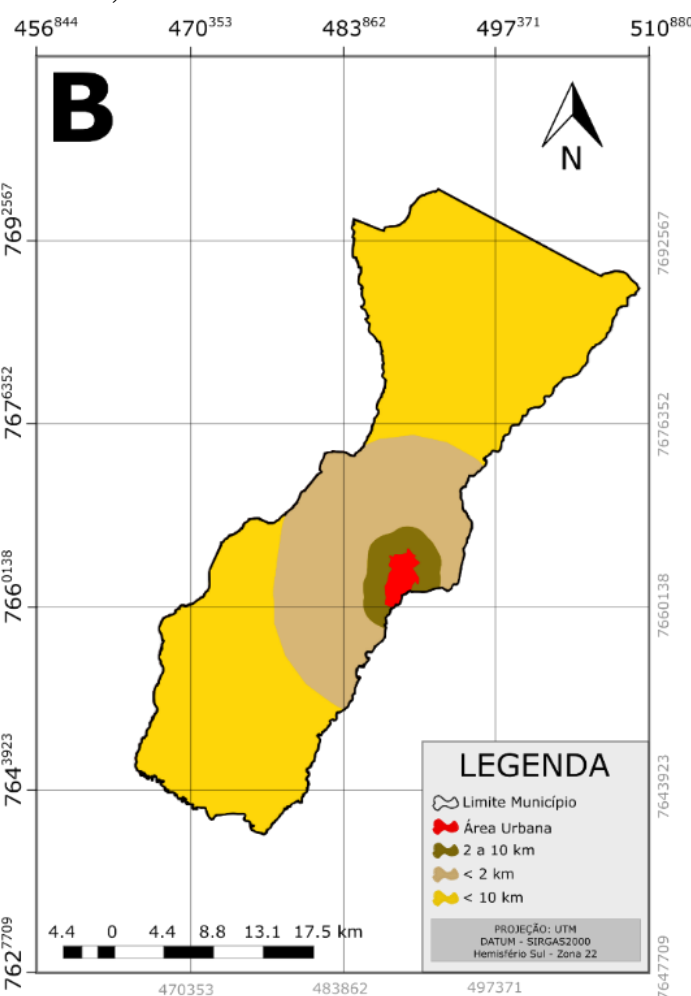

Figura 5 - Mapas de aptidão logística para o Município de Mirandópolis-SP. A) refere-se às distâncias a partir da malha viária. B) às distâncias até o centro de coleta.

Tabela 4 - Pesos de cada parâmetro obtidos a partir de diferentes fontes consultadas.

\begin{tabular}{c|c|c|c|c|c}
\hline Fonte & Declividade & Tipo de Solo & Uso do Solo & $\begin{array}{c}\text { Distância do } \\
\text { Centro de } \\
\text { Coleta }\end{array}$ & $\begin{array}{c}\text { Distância de } \\
\text { Rodovias }\end{array}$ \\
\hline Spigolon et al. (2015) & 0,1796 & 0,1078 & 0,3535 & 0,1796 & 0,1796 \\
\hline Weber \& Hasenack (2000) & 0,4434 & 0,3168 & 0,1274 & 0,0553 & 0,0571 \\
\hline Schmidt (2017) & 0,3860 & 0,2807 & 0,1404 & 0,0702 & 0,1228 \\
\hline Samizava et al. (2008) & 0,4720 & 0,1995 & - & 0,2301 & 0,0984 \\
\hline Feo \& Gisi (2014) & - & - & 0,7071 & - & 0,2929 \\
\hline Guiqin et al. (2009) & 0,2128 & - & 0,5423 & 0,1838 & 0,0611 \\
\hline Gbanie et al. (2013) & 0,1183 & 0,0969 & 0,4461 & 0,2071 & 0,1315 \\
\hline Motlagh \& Sayadi (2015) & 0,0893 & 0,3321 & 0,2607 & 0,1857 & 0,1321 \\
\hline Mohd Din et al. (2008) & 0,0579 & 0,2635 & 0,5409 & - & 0,1377 \\
\hline Sener et al. (2010) & 0,0589 & - & 0,5552 & 0,1948 & 0,1911 \\
\hline Gorsevski et al. (2012) & 0,1015 & - & 0,3441 & 0,5545 & - \\
\hline Moeinaddini et al. (2010) & 0,0912 & 0,1129 & 0,2314 & 0,3952 & 0,1694 \\
\hline Média dos Pesos Obs. & $\mathbf{0 , 2 0 1 0}$ & $\mathbf{0 , 2 1 3 8}$ & $\mathbf{0 , 3 8 6 3}$ & $\mathbf{0 , 2 2 5 6}$ & $\mathbf{0 , 1 4 3 1}$ \\
\hline Pesos finais normalizados & $\mathbf{0 , 1 7 1 8}$ & $\mathbf{0 , 1 8 2 8}$ & $\mathbf{0 , 3 3 0 2}$ & $\mathbf{0 , 1 9 2 9}$ & $\mathbf{0 , 1 2 2 3}$ \\
\hline
\end{tabular}

Os critérios de ponderação contidos nas tabelas 2, 3 e 4 foram então inseridos em um algoritmo em Linguagem Espacial para Geoprocessamento Algébrico (LEGAL), onde foram atribuídos os pesos máximo e mínimo de cada classe (Tabela 5) para proporcionar a análise espacial de geração do mapa de aptidão geral da área.

Posteriormente, sobre o mapa de aptidão geral foram feitos os recortes das áreas restritivas (áreas inaptas) e então gerado o mapa final de aptidão das áreas do município quanto a instalação do aterro sanitário. 
Tabela 5 - Ponderações de valores das classes utilizadas para a classificação de aptidão.

\begin{tabular}{|c|c|c|c|c|}
\hline \multicolumn{2}{|r|}{ Parâmetro } & Peso do Parâmetro & Classes & Valor da Classe \\
\hline \multirow{10}{*}{ 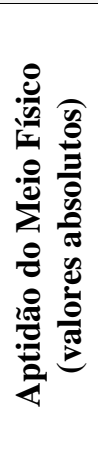 } & \multirow{3}{*}{ Declividade } & \multirow{3}{*}{0,1718} & $<2 \%$ & 2 \\
\hline & & & $2 \%-10 \%$ & 3 \\
\hline & & & $>10 \%$ & 0 \\
\hline & \multirow{5}{*}{$\begin{array}{l}\text { Tipo de Solo/ } \\
\text { Associações }\end{array}$} & \multirow{5}{*}{0,1828} & Neossolos & 1 \\
\hline & & & Neossolos + Argissolos & 1,5 \\
\hline & & & Argissolos & 2 \\
\hline & & & Argissolos + Latossolos & 2,5 \\
\hline & & & Latossolos & 3 \\
\hline & \multirow[t]{2}{*}{ Uso do Solo } & \multirow[t]{2}{*}{0,3302} & $\begin{array}{l}\text { Vegetação Arbórea } \\
\text { (grande porte) }\end{array}$ & 1 \\
\hline & & & Outros & 3 \\
\hline \multirow{4}{*}{ 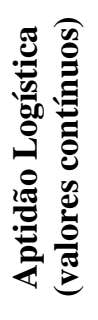 } & \multirow{2}{*}{$\begin{array}{c}\text { Distância Centro de } \\
\text { Coleta } \\
\left(\text { Dist }_{\text {urb }}\right)\end{array}$} & \multirow{2}{*}{0,1929} & $\geq 2.000 \mathrm{~m}$ & 3 (máx) \\
\hline & & & $\geq 10.000 \mathrm{~m}$ & 0 (min) \\
\hline & \multirow{2}{*}{$\begin{array}{l}\text { Distância de } \\
\text { Rodovias } \\
\text { (Dist }_{\text {rod }} \text { ) }\end{array}$} & \multirow{2}{*}{0,1223} & $\geq 75 \mathrm{~m}$ & 3 (máx) \\
\hline & & & $\geq 2.000 \mathrm{~m}$ & $0(\min )$ \\
\hline
\end{tabular}

\section{RESULTADOS E DISCUSSÃO}

Nas figuras 6 e 7 são apresentados como resultado os mapas de áreas restritivas (Figura 6a), de aptidão física/logística (Figura 6b), e de aptidão final (Figura 7) para instalação de aterro sanitário no município de Mirandópolis-SP.

Observa-se na figura $6 a$ que, com base unicamente nas informações técnicas e de acesso público gratuito, foi possível identificar e excluir aproximadamente $61 \%$ das áreas do município, uma vez que são categoricamente inaptas a este tipo de empreendimento. Como resultado, ao associar os mapas de restrição (Figura 6a) ao mapa de aptidão física/logística (Figura 6b) obteve-se o mapa de aptidão final onde estão (Figura 7), de forma escalada, as melhores áreas para instalação de um aterro sanitário.
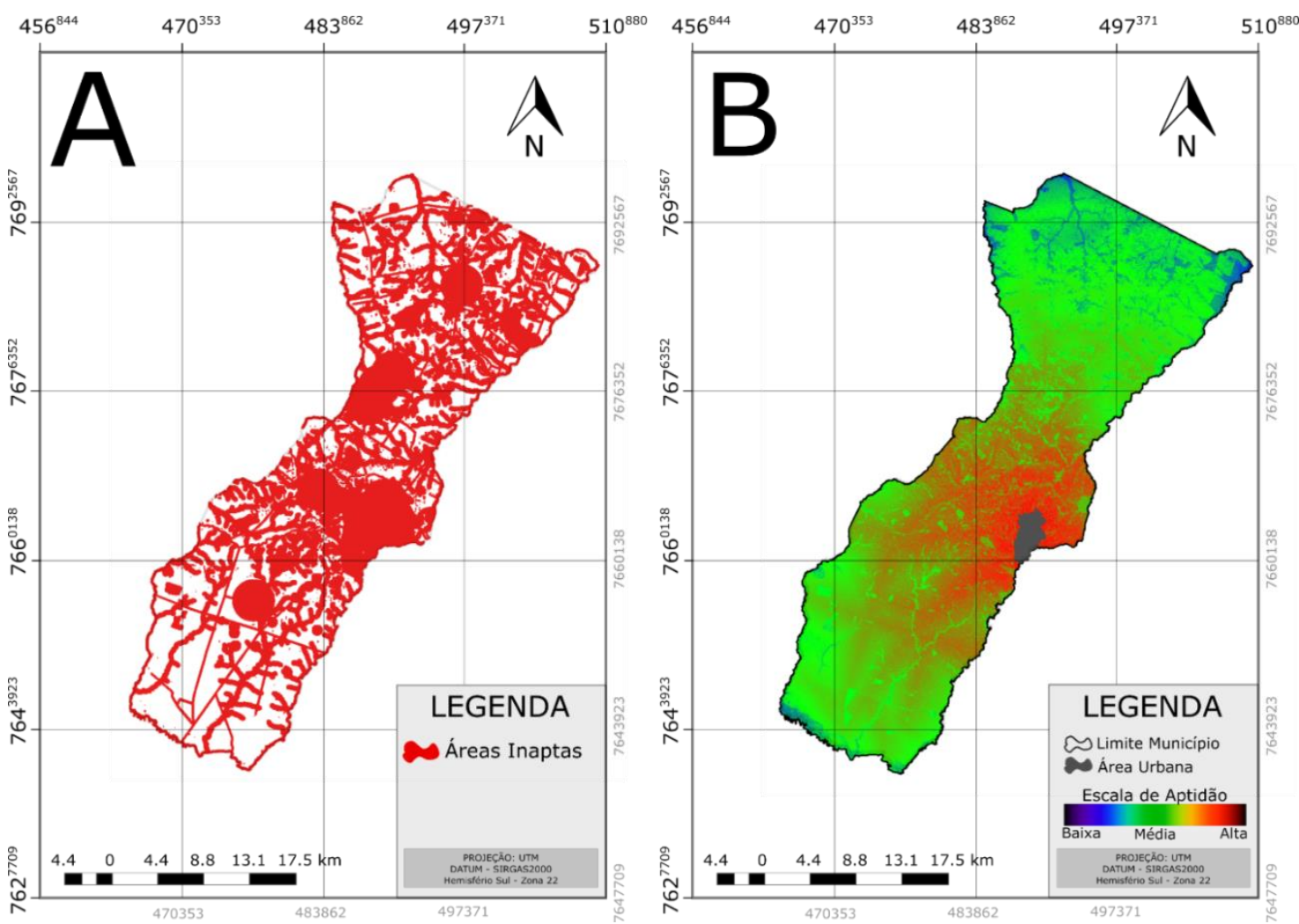

Figura 6 - Mapa de áreas restritivas (A) e de aptidão física/logística (B) para instalação de aterro sanitário no município de Mirandópolis-SP. 
A figura 7 delimita e limita áreas em potencial para o empreendimento, sugerindo locais para a implementação de aterros sanitários com foco no melhor custo benefício possível para a administração pública e atendendo, ainda, às normas vigentes no país.

A partir do mapa de aptidão final (Figura 7) ainda cabem algumas observações gerais de decisão, como por exemplo a direção dos ventos. Assim, considerando que a direção dos ventos na região tem predominância no sentido SE-NO (SÃO PAULO, 2012), haveria poucas áreas de exclusão, uma vez que há poucas áreas aptas à sudeste do perímetro urbano de Mirandópolis.

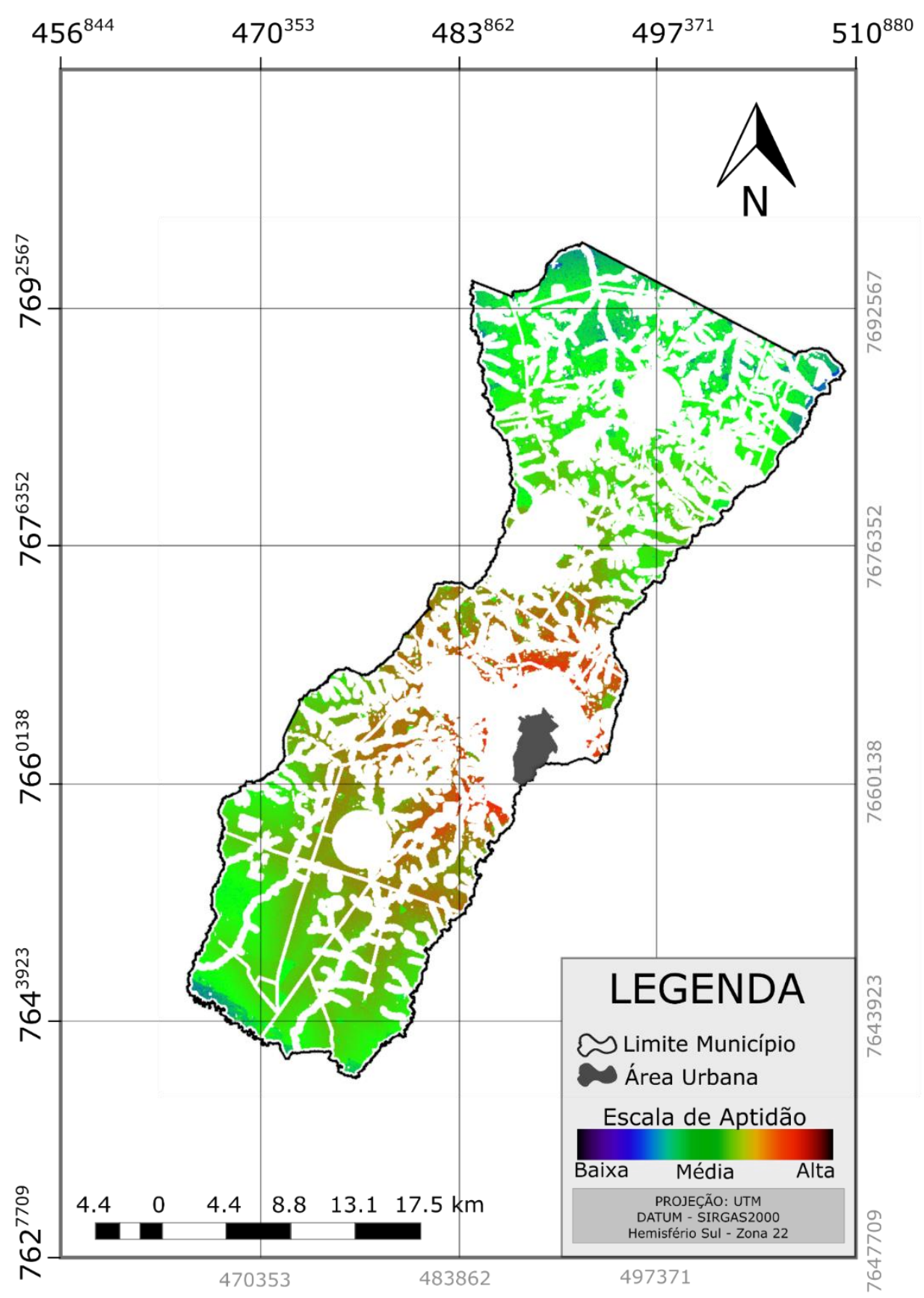

Figura 7 - Mapa final de aptidão para instalação de aterro sanitário no município de Mirandópolis-SP.

Por fim, tendo em vista a quantidade escassa de informações referente à permeabilidade do solo e à profundidade do nível freático tanto em bancos de dados públicos tradicionais, como o SIAGAS (CPRM, 2019), como na base de dados fornecida pela Prefeitura Municipal de Mirandópolis, e considerando que tais critérios dependem de informações locais cuja relevância está relacionada à fase de definição final da área escolhida e elaboração de projeto de construção do aterro sanitário, fica como última análise restritiva, dentro das áreas de interesse (definidas como aptas) a avaliação a campo dessas duas variáveis. 


\section{CONCLUSÕES}

As análises realizadas verificam que menos de $40 \%$ da área total do município é apta para a instalação de aterro sanitário, e as áreas mais adequadas se encontram no entorno do perímetro urbano, especialmente a nordeste e sudoeste deste. A abordagem adotada se baseia em dados e informações digitais facilmente acessíveis e de obtenção rápida. Tal quadro, associado a técnicas digitais de análise espacial, proporcionou grande agilidade ao processo.

Além disso, devido ao caráter da abordagem, o processo pode ser replicado em qualquer municipalidade em que haja a necessidade de avaliação de uma área adequada para a instalação de aterro sanitário visando o custo benefício, desde que esta possua os mesmos dados utilizados ou sugeridos no estudo.

Deve ser notado, no entanto, que o processo abordado se trata de uma avaliação preliminar, e para a decisão final quanto à instalação de um aterro sanitário, as áreas previamente selecionadas como as mais aptas devem ser submetidas a outras verificações, como quanto à profundidade do nível freático e à permeabilidade do solo, além de serem consideradas outras questões específicas como, por exemplo, o tamanho da área, a vida útil, e outras possíveis demandas da localidade

\section{REFERÊNCIAS}

ABNT-ASSOCIAÇÃO BRASILEIRA DE NORMAS TÉCNICAS. NBR 13.896 - Aterro de resíduos não perigosos: critérios para projeto, implantação e operação, 1997.

BING, X.; BLOEMHOF, M. J.; RAMOS, T. R. P.; BARBOSAPOVOA, A. P.; WONG, C. Y.; VORST, J.G.A.J. Research challenges in municipal solid waste logistics management. Waste Management, v. 48, p. 584-592, 2016.

BRASIL. Acesso à Informação: Arquivos Shapefile Gasodutos Brasil. Disp. em: https://goo.gl/TfrUCB. Acessado em: 04dez2017.

BRASIL. Lei $\mathrm{n}^{\circ} \mathbf{1 2 3 0 5}$, de 2 de agosto de 2010. Institui a Política Nacional de Resíduos Sólidos. Disp. em: http://www.planalto.gov.br/ccivil_03/_ato2007-

2010/2010/lei/112305.htm. Acessado em 12jun2018.

CAMARA G.; SOUZA, R.C.M.; FREITAS, U.M.; GARRIDO J.; MITSUO, F. SPRING: Integrating remote sensingand GIS by object-oriented data modelling. Computers \& Graphics, v. 20, n. 3, p. 395-403, 1996.

CHANG, N.; PARVATHINATHAN, G.; BREEDEN J. B. Combining GIS with fuzzy multicriteria decision-making for landfill siting in a fast-growing urban region. Journal of Environmental Management, v. 87, p. 139-153, 2008.

CPRM - SERVIÇO GEOLÓGICO DO BRASIL. Sistema de Informações de Águas Subterrâneas - SIAGAS. Disp. em: http://siagas.cprm.gov.br. Acessado em: 06out2019.

DEUS, R.M.; BATTISTELLE, R.A.G.; SILVA, G.H.R. Current and future environmental impact of household solid waste management scenarios for a region of Brazil: carbon dioxide and energy analysis. Journal of Cleaner Production, v. 155, n. 1, p. 218-228, 2017.

EASTMAN, J.R. Multi-criteria evaluation and GIS. In: LONGLEY, P. A.; GOODCHILD, M. F.; MAGUIRE, D. J. \& RHIND, D. W. (Ed.). Geographical Information Systems: Principles, Techniques, Applications and Management. Chichester, New York: Wiley, p. 493-502, 2005

EASTMAN, J.R.; JIN, W., PETER, A.K.; KYEM, TOLEDANO, J. Raster Procedure for Multi-Criteria/Multi-Objective Decisions. Photogrammetric Engineering \& Remote Sensing, v. 61, n. 5, p. 539-547, 1995.

ERIKSSON, O.; REICH, M.C.; FROSTELL, B.; BJÖRKLUND, A.; ASSEFA, G.; SUNDQVIST, J.O.; GRANATH, J.; BAKY, A.; THYSELIUS, L. Municipal solid waste management from a systems perspective. Journal of Cleaner Production, v. 13, n. 3, p. 241-252, 2005.

FEO, G. \& GISI, S. Using MCDA and GIS for hazardous waste landfill siting considering land scarcity for waste disposal. Waste Management, v. 34, n. 11, p. 2225-2238, 2014.

FERREIRA, E.M.; BARROS, R.T.V.; SOVIAR, J. Brazilian waste management: Belo Horizonte's case of study of sustainable management. Procedia Engineering, v. 192, p. 171-176, 2017.

GBANIE, S.P.; TENGBE, P.B.; MOMOH, J.S.; MEDO, J.; KABBA, V.T.S. Modelling landfill location using Geographic Information Systems (GIS) and Multi-Criteria Decision Analysis (MCDA): Case study Bo, Southern Sierra Leone. Applied Geography, v. 36, p. 3-12, 2013.

GORSEVSKI, P.V.; DONEVSKA, K.R.; MITROVSKI, C.D.; FRIZADO, J.P. Integrating multi-criteria evaluation techniques with geographic information systems for landfill site selection: A case study using ordered weighted average. Waste Management, v.32, n. 2, p. 287-296, 2012

GUIQIN, W.; LI, Q.; GUOXUE, L.; LIJUN, C. Landfill site selection using spatial information technologies and AHP: A case study in Beijing, China. Journal of Environmental Management, v. 90, n. 8, p. 2414-2421, 2009.

HOORNWEG, D. \& BHADA-TATA, P. What a waste: A global review of solid waste management. Washington: World Bank, 2012.

IBGE-INSTITUTO BRASILEIRO DE GEOGRAFIA E ESTATÍSTICA. Cidades. Disp. em: http://cod.ibge. gov.br/2VIBX. Acessado em: 02nov2017.

INPE-INSTITUTO NACIONAL DE PESQUISAS ESPACIAIS. Catálogo de imagens. Disponível em: http://www.dgi.inpe.br/catalogo/.

INPE-INSTITUTO NACIONAL DE PESQUISAS ESPACIAIS. Programa. Disp. em: http://www.dpi.inpe.br/spring/ portugues/download.php. Acessado em 03mar2018.

JAXA. Dataset: PRISM, JAXA 2008. Retrieved from ASF 21 Setembro 2018.

KAHRAMAN, C.; CEBI, S.; ONAR, S.C.; OZTAYSI, B. A novel trapezoidal intuitionistic fuzzy information axiom approach: An application to multicriteria landfill site selection. Engineering Application of Artificial Intelligence, v, 67, p. 157-172, 2018.

KHAN, M.; VAEZI, M.; KUMAR A. Optimal siting of solid waste-to-value-added facilities through a GIS-based assessment. Science of The Total Environment, v. 610-611, n. 1, p. 1065-1075, 2018.

KIKUCHI, R. \& GERARDO, R. More than a decade of conflict between hazardous waste management and public resistance: a case study of NIMBY syndrome in Souselas (Portugal). Journal of Hazardous Materials, v. 172, n. 2-3, p. 1681-1685, 2009.

LELLA, J.; MANDLA, V.R.; ZHU, X. Solid waste collection/transport optimization and vegetation land cover estimation using GIS: A case study of a proposed smart-city. 
Sustainable Cities and Society, v. 35, p. 336-349, 2017.

MOEINADDINI, M.; KHORASANI, N.; DANEHKAR, A.; DARVISHSEFAT, A.A.; ZIENALYAN, M. Siting MSW landfill using weighted linear combination and analytical hierarchy process (AHP) methodology in GIS environment (case study: Karaj). Waste Management, v. 30, n. 5, p. 912 920, 2010.

MOHD DIN, M.A.; JAAFAR, W.Z.W.; OBOT, R.M.M.; HUSSIN, M.A.W. How GIS can be a useful tool to deal with landfill site selection. In: GISIDEAS - INTERNATIONAL SYMPOSIUM ON GEOINFORMATICS FOR SPATIAL INFRASTRUCTURE DEVELOPMENT IN EARTH AND ALLIED SCIENCES, 2008, Hanoi, Vietnam. Conference paper... Hanoi.

MONTAÑO, M.; RANIERI, V.E.L.; SCHALCH, V.; FONTES, A.T.; CASTRO, M.C.A.A.; SOUZA, M.P. Integração de critérios técnicos, ambientais e sociais em estudos de alternativas locacionais para a implantação de aterro sanitário. Engenharia Sanitária Ambiental, v. 17, n. 1, p. 61-70, 2012.

MOTLAGH, Z.K. \& SAYADI, M.H. Siting MSW landfills using MCE methodology in GIS environment (case study: Birjand plain, Iran). Waste Management, v. 46, p. 322-337, 2015.

RAGHAVAN, K.; MANDLA, V.R.; FRANCO, S. Influence of urban areas on environment: Special reference to building materials and temperature anomalies using geospatial technology. Sustainable Cities and Society, v. 19, p. 349-358, 2015.

SAAEM-SERVIÇO AUTÔNOMO DE ÁGUA E ESGOTO DE MIRANDÓPOLIS. Plano de Saneamento Básico Municipal. Mirandópolis, 2014.

SAATY, T. L. A scaling method for priorities in hierarchical structures. Journal of Mathematical Psychology, v. 15, n. 3, p. 234-28, 1977.

SAMIZAVA, T.M.; KAIDA, R.H.; IMAI, N.N.; NUNES, J.O.R. SIG aplicado à escolha de áreas potenciais para instalação de aterros sanitários no município de Presidente Prudente - SP. Revista Brasileira de Cartografia, v. 1, n. 60, p. 43-55, 2008.

SÃO PAULO. Atlas Eólico do Estado de São Paulo. São Paulo: Governo do Estado de São Paulo, Secretaria de Energia, 135 p., 2012.

SÃO PAULO. Caderno Resíduos Sólidos. 2.ed. São Paulo: Governo do Estado de São Paulo, Secretaria de Meio Ambiente, Coordenadoria de Planejamento Ambiental, 164 p., 2013.

SÃO PAULO. Plano de resíduos sólidos do estado de São Paulo. São Paulo: Governo do Estado de São Paulo, Secretaria do Meio Ambiente do Estado de São Paulo, Coordenadoria de Planejamento Ambiental, CETESB, 350 p., 2014.
SÃO PAULO. Procedimentos para implantação de aterro sanitário em valas. São Paulo: Governo do Estado de São Paulo, Secretaria de Meio Ambiente, 34 p., 2005.

SCHMIDT, T. Seleção de área e dimensionamento de aterro sanitário para o consórcio público intermunicipal para assuntos estratégicos do G8 - Cipae G8. Lajeado, 2017, 146 p. Monografia (Graduação em Engenharia Ambiental) Universidade do Vale do Taquari.

SEADE. Perfil dos Municípios Paulistas: Mirandópolis Governo do Estado de São Paulo. Disp. em: http://www.perfil.seade.gov.br/. Acessado em: 08dez2017.

SENER, S.; SENER, E.; NAS, B.; KARAGUZEL, R. Combining AHP with GIS for landfill site selection: a case study in the Lake Beysehir catchment area (Konya, Turkey). Waste Management, v. 30, n. 11, p. 2037-2046, 2010.

SICAR-SISTEMA AMBIENTAL DE CADASTRO AMBIENTAL RURAL. Base de Downloads: São Paulo. Disp. em: http://www.car.gov.br/publico/municipios/down loads?sigla=SP. Acessado em: 23nov2017.

SNIS-SISTEMA NACIONAL DE INFORMAÇÕES SOBRE SANEAMENTO. Diagnóstico do manejo de Resíduos Sólidos Urbanos - 2016. Governo Federal: Ministério do Desenvolvimento Regional. Disp. em: http://www.snis.gov. br/diagnostico-residuos-solidos/diagnostico-rs-2016. Acessado em 23jan2019. (Planilhas).

SPIGOLON, L.T.G.; SOUZA, N.C., LAROCCA, A.P.C., GIANNOTTI, M.A., RUSSO, M.A.T., ALONSO, J.M. Seleção de áreas adequadas para a instalação de aterro sanitário utilizando SIG e análise multicritério - estudo de caso: UGRHI 5 (Piracicaba/Capivari/Jundiaí). In: SIMPÓSIO BRASILEIRO DE SENSORIAMENTO REMOTO, XVII, 2015, João Pessoa. Anais...João Pessoa: INPE, 2015.

SULLIVAN, D. Google Earth Pro, EContent, v. 32, n. 3, p. $16-$ $18,2009$.

TBG - TRANSPORTADORA BRASILEIRA GASODUTO BOLÍVIA-BRASIL. Faixa de Servidão. Disp. em: https://goo.gl/nuPmnp. Acessado em: 04dez2017.

WEBER, E. \& HASENACK, H. Avaliação de áreas para instalação de aterro sanitário através de análises em SIG com classificação contínua dos dados. In: CONGRESSO E FEIRA PARA USUÁRIOS DE GEOPROCESSAMENTO DA AMÉRICA LATINA, 6, 2000, Salvador. Conference paper... Salvador. Aceito em 18 de setembro de 2019 